Arq. Bras. Med. Vet. Zootec., v.71, n.1, p.177-186, 2019

\title{
Avaliação da produção lacrimal, pressão intraocular, retinografia e biometria ocular em bovinos das raças Nelore e Gir hígidos
}

\author{
[Evaluation of tear production, intraocular pressure, retinography and ocular biometry in \\ bovine Nelore and Gir breeds] \\ T.L. Morais, C.A. Neves ${ }^{*}$, N.C. Borges, A.M.V. Lima, L.F.L. Silva, \\ L.T.S. Castro, S.O. Espindola, P.H.J. Cunha \\ Universidade Federal de Goiás - Goiânia, GO
}

\begin{abstract}
RESUMO
No presente estudo, foram avaliados parâmetros oftalmológicos de 38 bovinos das raças Nelore ( $\mathrm{n}=19$; grupo GN) e Gir ( $n=19$; grupo GG), machos, com idade média de 15 meses. Os exames oftalmológicos realizados foram teste lacrimal de Schirmer 1 (TLS-1), tonometria de aplanação, retinografia e biometria por ultrassonografia em modo B. Os resultados do TLS-1, tonometria de aplanação e biometria ocular foram avaliados por meio da análise de variância (ANOVA), e as médias comparadas pelo teste de Tukey. Para o exame de retinografia, foi realizada a análise descritiva das estruturas anatômicas. O TLS-1 e a tonometria não diferiram $(\mathrm{P} \geq 0,05)$ nas comparações entre os olhos e entre os grupos raciais. A retinografia permitiu a visualização do disco óptico, dos vasos retinianos, do fundo tapetal e não tapetal, sem constatação de diferenças anatômicas entre as raças. Por meio da biometria ocular, foram observados valores maiores nos animais da raça Gir para as medidas do comprimento axial (CAx), da espessura da lente (EL) e da profundidade da câmara vítrea (CV), em ambos os eixos, horizontal (CAH) e vertical $(\mathrm{CAV})$. Os exames oftalmológicos realizados podem ser executados a campo sem comprometer o bemestar dos animais. Somente na ultrassonografia em modo B foram identificadas diferenças entre os bovinos das raças Nelore e Gir.
\end{abstract}

Palavras-chave: oftalmologia, retina, Schirmer, tonometria, ultrassonografia

\begin{abstract}
The ophthalmological parameters were evaluated of 38 male Nelore bovine $(n=19$, GN group) and Gir ( $n=19$, GG group), with 15 months age. The ophthalmological examinations by Schirmer 1 (TLS-1), aplanation tonometry, retinography and B-mode ultrasonography were done. The results of the TLS-1, aplanation tonometry and ocular biometry were evaluated by analysis of variance and means compared by the Tukey test. A descriptive analysis of the anatomical structures was performed for the retinography examination. TLS-1 and tonometry did not differ $(P \geq 0.05)$ in the comparisons between the eyes and between the groups. Retinography allowed the visualization of the optic disc, retinal vessels, tapetal and non-tapetal fundus, without any anatomical differences between the breed. In the ocular biometry, higher values were observed in Gir animals for measurements of axial length (CAx), lens thickness (EL) and depth of the vitreous chamber $(\mathrm{CV})$, both horizontal (CAH) and vertical (CAV) imaging planes. Ophthalmologic examinations can be performed on the field without compromising animal welfare. Differences between Nelore and Gir bovines were identified only by B-mode ultrasonography.
\end{abstract}

Keywords: ophthalmology, retina, schirmer, tonometry, ultrasonography

Recebido em 23 de agosto de 2017

Aceito em 22 de maio de 2018

*Autor para correspondência (corresponding author)

E-mail: carlaamorim.vet@gmail.com 


\section{INTRODUÇÃO}

A padronização das avaliações oculares, considerando-se as diferentes raças de bovinos, pode contribuir para o diagnóstico de oftalmopatias de importância econômica e zoonótica, pois variações anatômicas raciais são comuns e devem ser consideradas durante a avaliação oftálmica (Tektas et al., 2010).

Estudos realizados em matadouros indicaram incidência de $14,6 \%$ a $100 \%$ de lesões oculares, dependendo da idade e produtividade dos animais examinados (Potter et al., 2008). Os conhecimentos relacionados à anatomia, bem como as dimensões do olho, podem auxiliar no diagnóstico diferencial das oftalmopatias (Potter et al., 2008), e os valores basais dos parâmetros oculares em animais saudáveis são importantes para se estabelecerem intervalos de normalidade (Honsho et al., 2016).

Com o TLS-1, é possível mensurar a produção da porção aquosa da lágrima, o que auxilia no diagnóstico diferencial de oftalmopatias que cursam com alterações na produção lacrimal, tanto em humanos, quanto em animais (Oriá et al., 2014; Pietro et al., 2016). No entanto, existem poucos relatos para esse parâmetro em bovinos (Tofflemire et al., 2015; Pietro et al., 2016).

A mensuração da pressão intraocular (PIO) pelo método de tonometria é uma ferramenta essencial para o diagnóstico de doenças oculares como glaucoma e uveíte (Andrade et al., 2011). O Tono-Pen AVIA® é o instrumento de aplanação mais empregado, no entanto é bastante oneroso, o que restringe o seu uso em animais destinados à produção (Maggs et al., 2013).

O exame macroscópico da retina pode ser realizado por meio da retinografia e pode fornecer imagens de alterações retinianas, como o descolamento da retina, glaucoma e degeneração macular (Santos et al., 2012). Apesar de a morfologia macroscópica da retina bovina já ser descrita, existem poucos estudos avaliando cada raça especificamente (Pearce e Moore, 2013).

A ultrassonografia ocular tem sido utilizada, principalmente, quando há opacidade dos meios oculares (córnea, humor aquoso, lente e câmera vítrea), presente em enfermidades como ceratoconjuntivite infecciosa bovina, úlceras de córnea, ceratite, uveíte anterior e muitas doenças sistêmicas, como febre catarral maligna e diarreia viral bovina (Potter et al., 2008; Kassab, 2012). A ultrassonografia também é empregada na realização da biometria ocular, que pode ser utilizada no diagnóstico de alterações patológicas, como a atrofia ocular, microftalmia, pseudoexoftalmia e glaucoma congênito, além de ser de grande importância no planejamento cirúrgico de procedimentos, como a facectomia, e no descarte de alterações que impedem a cirurgia, como descolamentos de retina (Corrêa et al., 2014; Ferreira et al., 2014).

A escassez de referências sobre o tema, principalmente avaliando-se o animal vivo, associada com a importância que a bovinocultura representa no contexto mundial, justifica a realização de um estudo comparativo sobre os parâmetros oculares em bovinos hígidos de diferentes raças. Dessa forma, objetivou-se realizar avaliações oftalmológicas comparativas entre as raças de bovinos Nelore e Gir.

\section{MATERIAL E MÉTODOS}

O estudo foi desenvolvido após a aprovação da Comissão de Ética no Uso de Animais da Universidade Federal de Goiás (Ceua/UFG), registrado sob o número 025/2016. Foram utilizados 38 bovinos machos, hígidos, com idade média de 15 meses, sendo 19 animais da raça Nelore (GN) e 19 animais da raça Gir (GG), com pesos médios de $160 \pm 37,97 \mathrm{~kg}$ e $284 \pm 54,94 \mathrm{~kg}$, respectivamente. Durante o período experimental, os animais foram mantidos em pasto de Brachiaria decumbens, suplementados com sal mineral e água à vontade.

Os testes oftalmológicos realizados foram teste lacrimal de Schirmer-1 (TLS-1), tonometria de aplanação (Tono-Pen AVIA vet ${ }^{\circledR}$, Reichert, Nova York, Estados Unidos), retinografia (Clearview Fundus Camera - Opitibrand ${ }^{\mathrm{TM}}$, Colorado, Estados Unidos) e biometria (Logiq GE Healtchare®, Waukesha, Estados Unidos).

Para o TLS-1, seguiram-se as instruções de Tofflemire et al. (2015). Foram utilizadas tiras de papel-filtro estéreis padronizadas (Ophtalmos ${ }^{\circledR}$, São Paulo, Brasil), colocadas junto ao terço medial do saco conjuntival da pálpebra 
inferior do olho, durante um minuto. As pálpebras foram cuidadosamente mantidas fechadas, com o intuito de evitar movimentações excessivas das tiras. Em seguida, foi realizada a remoção do papel-filtro e imediata leitura da escala milimetrada de umidade presente na tira.

A tonometria de aplanação foi realizada logo após a citologia, de acordo com Ghaffari et al. (2011). O tonômetro foi posicionado com a base apoiada sobre o centro da córnea, sendo o exame executado por um único examinador. Antes do exame, foi instilada uma gota do colírio anestésico de cloridrato de proximetacaína $0,5 \%$ (Anestalcon, Alcon ${ }^{\circledR}$, São Paulo, Brasil) para a dessensibilização da superfície ocular. Para cada animal, foram realizadas cinco aferições consecutivas em cada olho, sendo calculada posteriormente a média das leituras adquiridas.

A avaliação retiniana foi realizada após a dilatação da pupila por meio da instilação de duas gotas de tropicamida $1 \%$ (Mydriacyl $^{\circledR}$, Alcon, São Paulo, Brasil). Após 30 minutos, foram realizadas fotografias de cada olho e as imagens comtemplavam todas as estruturas anatômicas de fundo de olho, como o disco óptico, os vasos retinianos, o fundo tapetal e não tapetal. Com o programa Clearview (Optical Imaging System, versão 4.5.8.0), avaliaram-se as imagens capturadas, as quais foram comparadas dentro dos grupos e entre eles.

A biometria ocular foi obtida pela ultrassonografia em modo $\mathrm{B}$, com transdutor linear com frequência de $12 \mathrm{MHz}$. Para a sua execução, foi instilada outra gota do colírio anestésico para a dessensibilização corneana. Foi preconizada a utilização de gel acústico estéril $\left(\right.$ Carbogel $^{\circledR}$, São Paulo, Brasil) e a proteção do transdutor com uma camada de gel recoberta por película aderente (Lusafilm R $105^{\circledR}$, Guarulhos, Brasil).

O exame ultrassonográfico foi executado por um único examinador. As medidas oculares foram realizadas em corte axial horizontal e vertical, com o transdutor posicionado no centro da córnea. Posteriormente, foram mensurados o comprimento axial (CAx - da camada epitelial da córnea à parede do fundo ocular), a profundidade da câmara anterior (CA - da camada endotelial da córnea à cápsula lenticular anterior ), a espessura da lente (EL - entre a cápsula lenticular anterior e posterior), a profundidade da câmara vítrea $(\mathrm{CV}$ - entre a cápsula lenticular posterior e a parede posterior do fundo ocular). Para cada animal, foram obtidas duas imagens de corte axial horizontal $(\mathrm{CAH})$ e duas de corte axial vertical (CAV) e calculadas as médias dessas medidas, obtendo-se, assim, o valor médio de cada corte.

Após o exame, foi feita limpeza dos olhos dos animais com solução fisiológica $\quad 0,9 \%$ (Equiplex ${ }^{\circledR}$, Aparecida de Goiânia, Brasil), sendo promovida uma leve pressão da solução em contato com a córnea para a retirada do excesso de gel acústico. Os dados e as imagens foram armazenados em arquivos para posterior análise estatística.

Foram calculadas as médias e os desvios-padrão dos dados encontrados no TLS-1, na tonometria e na biometria ocular e, posteriormente, estes foram analisados por análise de variância (ANOVA), empregando-se o teste de Tukey, por meio do software GraphPad Instat 3.0 (GraphPad Software Inc, San Diego, CA, USA) com grau de significância de 5\%. Para o exame de retinografia, foi realizada a análise descritiva das estruturas anatômicas, na qual as variáveis observadas foram a vasculatura retiniana, a morfologia do nervo óptico e as características das áreas tapetal e não tapetal.

\section{RESULTADOS}

Os valores obtidos durante a realização do TLS-1 e da tonometria dos animais das raças Nelore e Gir estão descritos na Tab. 1. Não foram observadas diferenças estatísticas $(\mathrm{P}>0,05)$ nos valores do TLS-1 entre as raças Nelore e Gir. Os valores médios de pressão intraocular encontrados no exame de tonometria também não evidenciaram diferença entre os grupos raciais e entre os olhos de cada grupo.

Por meio da retinografia, foi possível a visualização das estruturas de fundo de olho, que consistem de um tapetum lucidum ou fundo tapetal, um tapetum nigrum ou fundo não tapetal, um disco óptico e vasos sanguíneos da retina. Nos animais examinados, não foram observadas diferenças anatômicas entre as raças. 


\section{Morais et al.}

Tabela 1. Média e desvio-padrão dos resultados do teste lacrimal de Schirmer (TLS-1) e da pressão intraocular (PIO) em bovinos hígidos das raças Nelore (GN) e Gir (GG). Valores expressos em mm/min.

\begin{tabular}{|c|c|c|c|}
\hline & GN & GG & $\mathrm{P}$ \\
\hline \multicolumn{4}{|c|}{ TLS-1 } \\
\hline $\mathrm{OE}$ & $24,37 \pm 4,83$ & $24,68 \pm 6,49$ & 0,868 \\
\hline OD & $22,63 \pm 5,14$ & $23,74 \pm 5,47$ & 0,528 \\
\hline Média OE + OD & $23,50 \pm 5,00$ & $24,21 \pm 5,94$ & 0,582 \\
\hline \multicolumn{4}{|c|}{ Tonometria } \\
\hline $\mathrm{OE}$ & $11,72 \pm 2,03$ & $13,17 \pm 2,39$ & 0,051 \\
\hline OD & $12,00 \pm 2,34$ & $12,31 \pm 2,17$ & 0,691 \\
\hline Média OE + OD & $11,86 \pm 2,16$ & $12,74 \pm 2,29$ & 0,090 \\
\hline
\end{tabular}

$\mathrm{P}=$ probabilidade, $\mathrm{OE}=$ olho esquerdo, $\mathrm{OD}=$ olho direito.

O fundo tapetal (Fig. 1) encontra-se localizado na porção superior da retina e apresentou uma coloração azul-esverdeada em todos os animais, entretanto, quando a luz do aparelho era incidida com maior intensidade, a coloração era alterada para um tom amarelado. Não se verificou alteração na refletividade do fundo tapetal em ambos os grupos e observou-se aparência manchada ou granular.

Em todos os animais verificou-se presença de manchas enegrecidas na periferia de toda a área tapetal (Fig. 2). O fundo não tapetal apresentou a coloração marrom-escura.

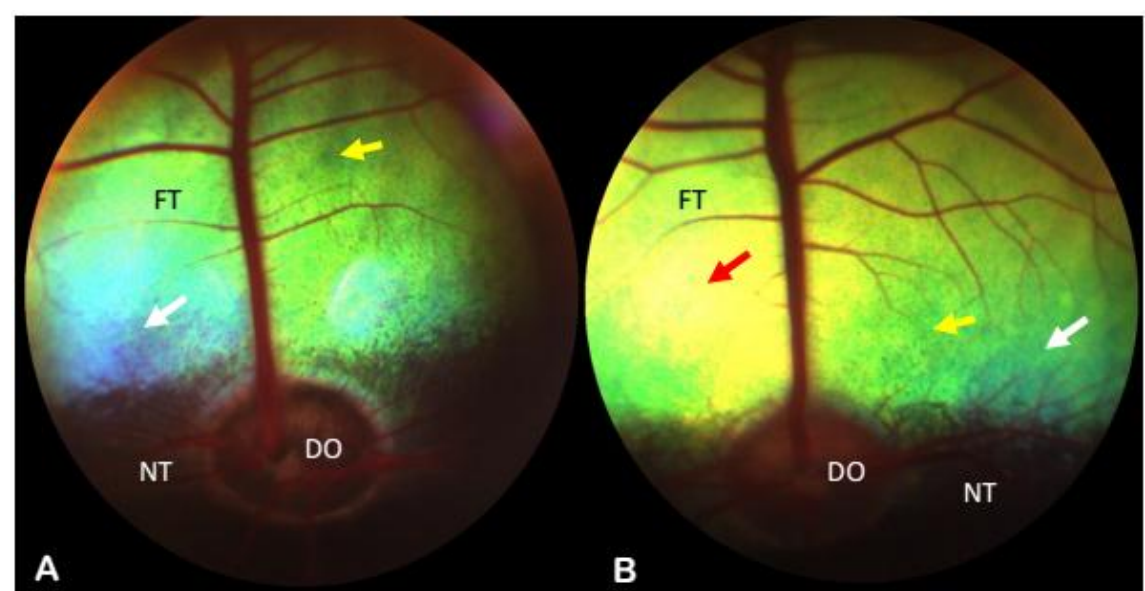

Figura 1. Retinografia de olhos esquerdos de bovinos das raças Nelore (A) e Gir (B). Observa-se fundo tapetal (FT) e não tapetal (NT), disco óptico (DO) e vasos retinianos. O fundo tapetal apresenta coloração azulada em sua porção ventral (seta branca), esverdeada em sua porção dorsal, e uma região amarelada onde houve maior incidência de luz (seta vermelha). No fundo tapetal, também é possível observar manchas escuras com aspecto pontilhado (seta amarela). 


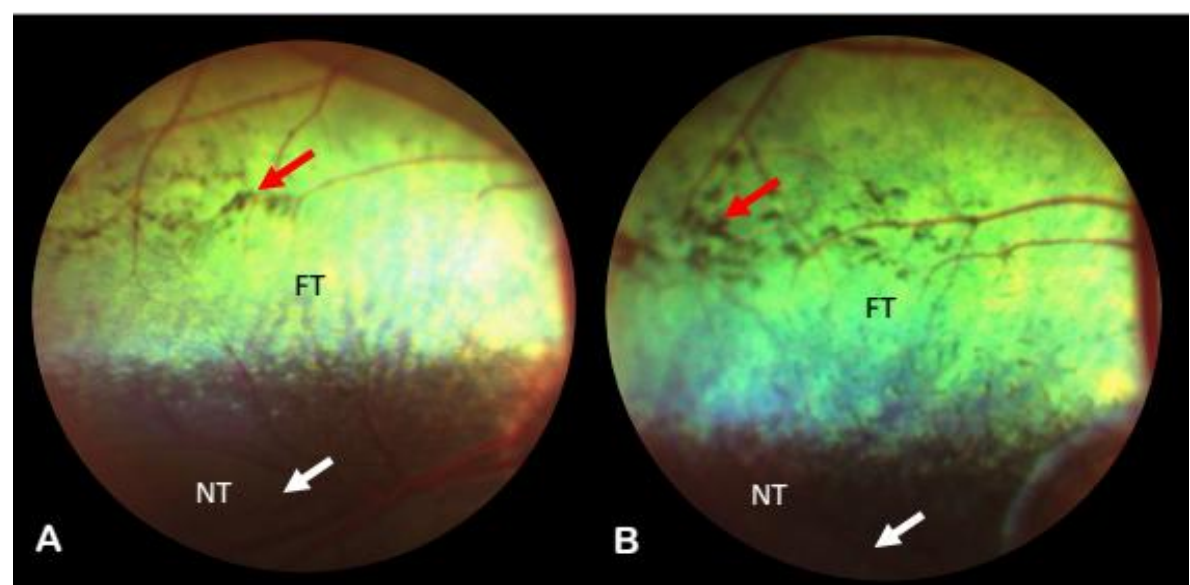

Figura 2. Retinografia de olhos esquerdos de bovinos das raças Nelore (A) e Gir (B). Presença de manchas enegrecidas (seta vermelha) na margem nasal da região tapetal (FT). O fundo não tapetal (NT) com cor marrom-escura (seta branca).

O disco óptico (Fig. 3) estava sempre inserido na área não tapetal, logo abaixo da região de transição do fundo tapetal para o não tapetal. Apresentou formato ovalado, com diâmetro horizontal maior que o vertical, a coloração variava entre tonalidades de laranja e marrom, e com borda bem definida de tonalidade esbranquiçada. Notou-se ainda um ponto esbranquiçado no centro do disco óptico.

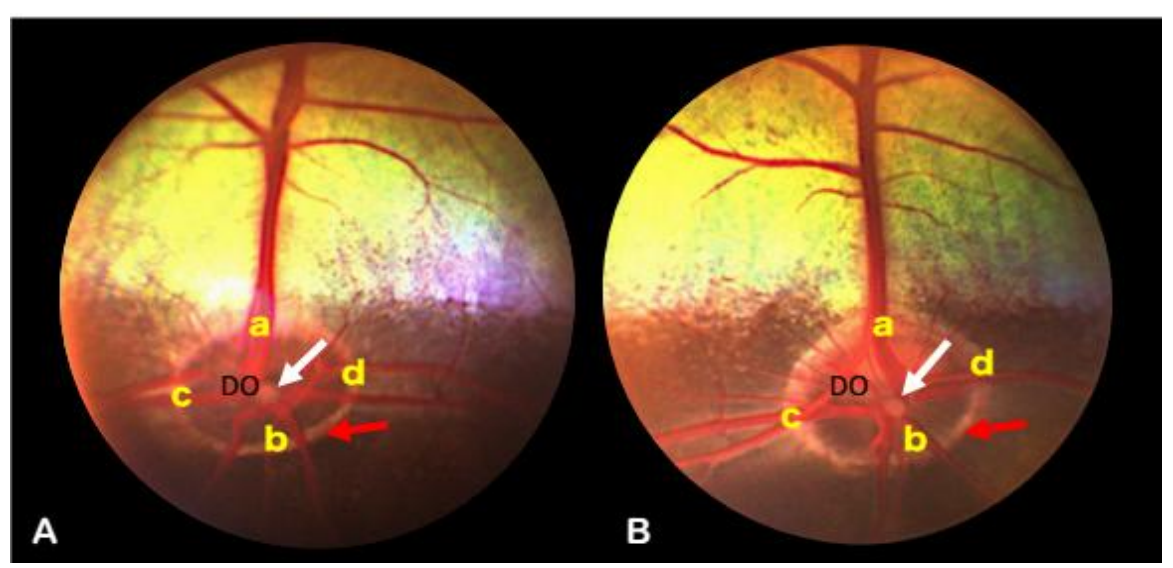

Figura 3. Retinografia de olhos esquerdos de bovinos das raças Nelore (A) e Gir (B). Disco óptico (DO) localizado na região não tapetal, com formato ovalado, diâmetro horizontal maior que o vertical, coloração entre tonalidades de laranja e marrom, com borda esbranquiçada bem definida (seta vermelha). Presença de um ponto central esbranquiçado (seta branca). Padrão vascular retiniano do tipo holangiótico. Presença de quatro pares primários de vasos emergindo do DO, nas regiões dorsal (a), ventral (b), ventromedial (c) e ventrolateral (d).

O padrão vascular retiniano visualizado foi do tipo holangiótico, formado por vasos que atravessam a superfície da retina. Verificou-se a presença de quatro pares primários de vasos que convergiam sobre o disco óptico, sendo um na região dorsal, um ventral, um ventrolateral e um ventromedial. Os vasos primários apresentavamse entrelaçados, principalmente o par de vasos dorsal.
Por meio da ultrassonografia em modo B (Fig. 4), visualizou-se a córnea como duas linhas convexas ecogênicas com um espaço anecoico estreito, a câmara anterior como uma área anecoica, imediatamente posterior à córnea. A íris e o corpo ciliar foram visualizados como estruturas moderadamente ecogênicas, adjacentes à câmera anterior. A cápsula anterior da lente mostrou-se como uma linha hiperecoica convexa, 
separada de uma linha hiperecoica côncava (cápsula posterior) pelo conteúdo lenticular. A câmara vítrea apresentou-se anecoica e precedeu o complexo retina-coroide-esclera, o qual foi visibilizado como uma linha hiperecogênica côncava.
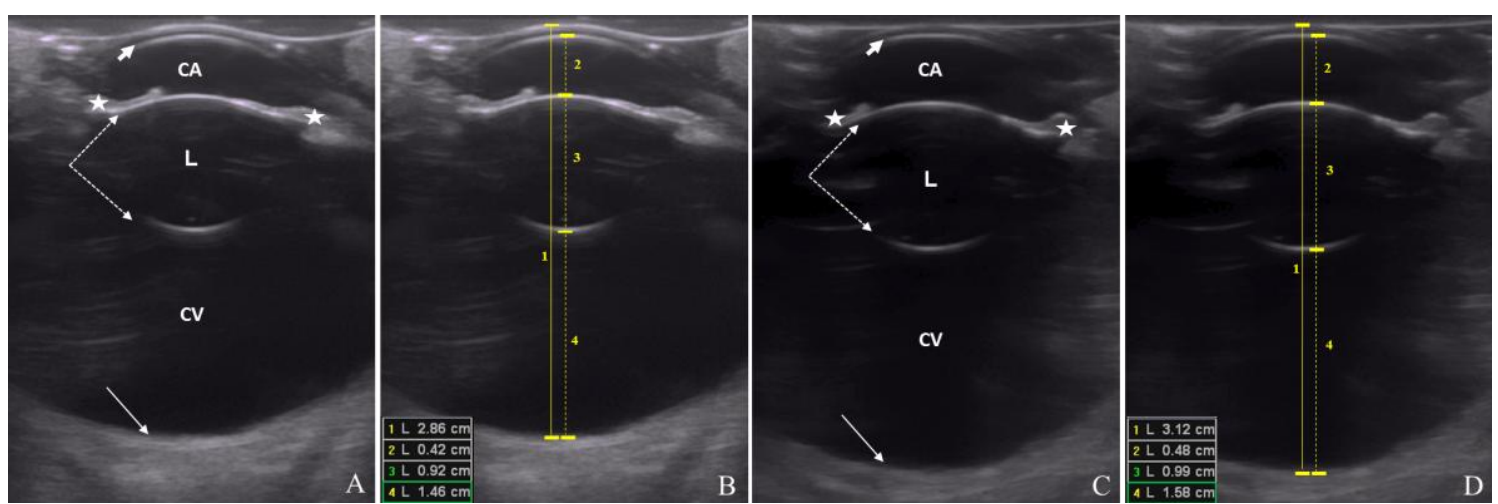

Figura 4. Ultrassonografia em modo B, cortes axiais verticais de olhos direitos de bovinos das raças Nelore (A e B) e Gir (C e D). A e B: Camadas hiperecoicas separadas por camada anecoica da córnea (seta curta), câmara anterior, lente e câmara vítrea anecoicas (CA, L e CV), cápsulas anterior e posterior da lente vistas como linhas curvilíneas (setas pontilhadas), íris e corpo ciliar hiperecoicos e espessos (asteriscos), parede posterior hiperecoica (seta larga). C e D: As linhas indicam a biometria ocular, sendo a linha contínua a medida de comprimento axial (1), e as linhas pontilhadas as medidas da câmara anterior (2), espessura lenticular (3) e profundidade da câmara vítrea (4). Frequência de $12 \mathrm{MHz}$.

Os resultados do exame de biometria ocular (Fig. 4) encontram-se expressos em média e desviopadrão (Tab. 2). Constatou-se diferença $(\mathrm{P}<0,05)$ entre grupos para as medidas CAx, EL e CV, em ambos os eixos (CAH e CAV). No entanto, não houve diferença $(\mathrm{P}>0,05)$ entre os olhos direito $\mathrm{e}$ esquerdo, entre os eixos e para a medida CA.

Tabela 2. Média e desvio-padrão dos resultados do exame de biometria ultrassonográfica realizada com transdutor linear com frequência de $12 \mathrm{MHz}$, expressos em centímetros $(\mathrm{cm})$, em bovinos hígidos das raças Nelore e Gir

\begin{tabular}{ccccccc} 
& & \multicolumn{2}{c}{ Grupo Nelore } & \multicolumn{2}{c}{ Grupo Gir } & \multirow{2}{*}{$\mathrm{P}$} \\
\cline { 3 - 6 } & & OE & OD & OE & OD & \\
\hline \multirow{4}{*}{$\mathrm{CAH}$} & $\mathrm{CAx}$ & $2,88 \mathrm{Aa} \pm 0,04$ & $2,89 \mathrm{Aa} \pm 0,05$ & $3,03 \mathrm{Ab} \pm 0,07$ & $3,03 \mathrm{Ab} \pm 0,08$ & $<0,0001$ \\
& $\mathrm{CA}$ & $0,44 \mathrm{Aa} \pm 0,03$ & $0,45 \mathrm{Aa} \pm 0,03$ & $0,45 \mathrm{Aa} \pm 0,03$ & $0,46 \mathrm{Aa} \pm 0,04$ & 0,132 \\
& $\mathrm{EL}$ & $0,93 \mathrm{Aa} \pm 0,02$ & $0,93 \mathrm{Aa} \pm 0,03$ & $0,97 \mathrm{Ab} \pm 0,03$ & $0,97 \mathrm{Ab} \pm 0,03$ & $<0,0001$ \\
& $\mathrm{CV}$ & $1,44 \mathrm{Aa} \pm 0,04$ & $1,44 \mathrm{Aa} \pm 0,04$ & $1,52 \mathrm{Ab} \pm 0,06$ & $1,52 \mathrm{Ab} \pm 0,06$ & $<0,0001$ \\
& $\mathrm{CAx}$ & $2,89 \mathrm{Aa} \pm 0,04$ & $2,89 \mathrm{Aa} \pm 0,04$ & $3,03 \mathrm{Ab} \pm 0,08$ & $3,03 \mathrm{Ab} \pm 0,07$ & $<0,0001$ \\
$\mathrm{CAV}$ & $\mathrm{CA}$ & $0,45 \mathrm{Aa} \pm 0,02$ & $0,44 \mathrm{Aa} \pm 0,03$ & $0,46 \mathrm{Aa} \pm 0,03$ & $0,46 \mathrm{Aa} \pm 0,04$ & 0,405 \\
& $\mathrm{EL}$ & $0,93 \mathrm{Aa} \pm 0,02$ & $0,93 \mathrm{Aa} \pm 0,02$ & $0,97 \mathrm{Ab} \pm 0,97$ & $0,97 \mathrm{Ab} \pm 0,03$ & $<0,0001$ \\
& $\mathrm{CV}$ & $1,44 \mathrm{Aa} \pm 0,04$ & $1,44 \mathrm{Aa} \pm 0,04$ & $1,52 \mathrm{Ab} \pm 0,06$ & $1,52 \mathrm{Ab} \pm 0,05$ & $<0,0001$ \\
\hline
\end{tabular}

Médias seguidas de letra maiúscula indicam diferença significativa entre os olhos pelo teste de Tukey com 5\% de probabilidade. Médias seguidas de letra minúscula indicam diferença significativa entre grupos experimentais pelo teste de Tukey com 5\% de probabilidade. $\mathrm{CAx}=$ comprimento axial, $\mathrm{CA}=$ profundidade da câmara anterior, $\mathrm{EL}=$ espessura da lente. $\mathrm{CV}=$ profundidade da câmara vítrea, $\mathrm{CAH}=$ eixo horizontal, $\mathrm{CAV}=$ eixo vertical, $\mathrm{OE}=$ olho esquerdo, $\mathrm{OD}=$ olho direito, $\mathrm{P}=$ probabilidade.

\section{DISCUSSÃO}

Os resultados da avaliação da produção lacrimal das raças Nelore $(23,50 \pm 5,00)$ e Gir $(24,21 \pm 5,94)$ foram semelhantes aos das raças Holandesa
$(20,00 \pm 5,20 \mathrm{~mm} / \mathrm{min}) \quad$ e Modicana $(20,00 \pm 5,20 \mathrm{~mm} / \mathrm{min})$. Similar ao presente trabalho, Pietro et al. (2016) não observaram diferenças na comparação entre os olhos e entre as raças em sua pesquisa. Resultados diferentes 
foram relatados por Wieser et al. (2013) e Beckwith-Cohen et al. (2014) ao avaliarem bovinos da raças Holandesa $(24,18 \pm 6,5 \mathrm{~mm} / \mathrm{min})$ e Austrian Spotted Mountain $(34,15 \pm 20,47 \mathrm{~mm} / \mathrm{min})$, respectivamente. No entanto, os autores utilizaram distintas metodologias, o que justifica as diferenças encontradas entre os resultados. Beckwith-Cohen et al. (2014) mantiveram as tiras na conjuntiva por 30 segundos, e Wieser et al. (2013) utilizaram tiras com comprimento maior $(10 \times 100 \mathrm{~mm})$ do que as utilizadas na presente pesquisa $(5 \times 40 \mathrm{~mm})$; devido a isso, os animais apresentaram uma produção lacrimal por minuto maior que a comportada pela tira.

Os valores de pressão intraocular (PIO) foram obtidos por meio do tonômetro de aplanação Tono-Pen AVIA vet ${ }^{\circledR}$, e as médias encontradas nos animais da raça Nelore $(11,86 \pm 2,16)$ e da raça Gir $(12,74 \pm 2,29)$ foram semelhantes entre os olhos (direito e esquerdo) e entre as raças avaliadas. Gum et al. (1998) obtiveram a média de $26,9 \pm 6,7 \mathrm{mmHg}$ da PIO das raças Jersey e Holandesa com o tonômetro Tono-Pen XL® e identificaram semelhanças significativas entre as médias entre os olhos e entre as raças. Andrade et al. (2011) avaliaram a PIO de bovinos utilizando o tonômetro de aplanação Tono-Pen XL ${ }^{\circledR}$ e obtiveram valores médios de $17,2 \pm 2,4 \mathrm{mmHg}$.

Os valores maiores encontrados nos resultados descritos por esses autores podem ser devido ao tipo de tonômetro utilizado, uma vez que o tipo e o modelo do tonômetro podem também influenciar nas medições de PIO (Gum et al., 1998). Andrade et al. (2011) compararam a PIO de equinos utilizando dois equipamentos diferentes, sendo os resultados do tonômetro de rebote Tono Vet ${ }^{\circledR}$ maiores $(30,04 \pm 3,19 \mathrm{mmHg})$ quando comparados aos do tonômetro de aplanação do Tono-Pen AVIA® $(23,10 \pm 4,01 \mathrm{mmHg})$. Este estudo evidencia que o tipo de tonômetro utilizado influenciou nos resultados encontrados, corroborando as informações encontradas na literatura. Todavia, não foram encontradas referências relacionadas ao uso do Tono-Pen AVIA ${ }^{\circledR}$ em bovinos, tampouco estudos com Tono-Pen AVIA vet巴.

O fundo tapetal nas raças Nelore e Gir estava localizado na área dorsal da retina e apresentou a coloração azul-esverdeada semelhante à identificada em outras espécies de bovinos Gelatt (2008). De acordo com Pearce e Moore (2013), nessa espécie, o fundo tapetal encontra-se localizado na região dorsal da retina e sua coloração pode variar de amarela até roxoazulada. No entanto, a cor do fundo tapetal pode ser influenciada por fatores como raça, idade, cor da pelagem e quantidade de pigmentação nos olhos e na pele (Ollivier et al., 2004). Os bovinos da raça Gir se distinguem pela pelagem vermelha ou amarela, e os Nelores pela pelagem brancocinza (Sarcinelli et al., 2007). Contudo, apesar das diferenças na pelagem das raças, não foram notadas diferenças na coloração do fundo tapetal visualizada entre os grupos.

A refletividade do fundo tapetal também pode ser avaliada durante o exame. As áreas com maior refletividade podem significar um afinamento da retina, enquanto as áreas com refletividade menor podem conter inflamação ou infiltrado celular retiniano ou sub-retiniano (Townsend, 2010). No entanto, no presente estudo, nenhuma alteração na refletividade foi encontrada, uma vez que foram utilizados animais hígidos.

A área não tapetal é uma região muito pigmentada e apresenta uma tonalidade marromescura (Gelatt, 2003), característica também constatada nos bovinos avaliados. Todavia, essa região pode apresentar áreas com coloração cinza a rosa, causadas por infiltrados celulares dentro da retina, ou no espaço sub-retiniano, ou em áreas com perda da pigmentação devido à degeneração ou atrofia da retina. Além disso, em bovinos, especialmente na raça Hereford, os colobomas da coroide são relativamente comuns e aparecem como divertículos no fundo do olho de coloração que varia do branco ao cinza (Townsend, 2010).

As manchas escuras pontilhadas com aspecto granulado visualizadas na área tapetal dos animais do presente estudo são chamadas de "estrelas de Winslow" (Stars of Winslow), as quais representam os vasos sanguíneos coroidais profundos atravessando o tapete e comunicandose com os coriocapilares (Gelatt, 2008). De acordo com Braekevelt (1986), melanócitos são encontrados progressivamente na periferia do fundo tapetal de bovinos, onde se misturam com as células dele. Por isso, é comum visualizar manchas de pigmentos amarronzados na região 
periférica do fundo tapetal de bovinos (Ollivier et al., 2004), como foram visualizadas em ambos os grupos.

O disco óptico de ruminantes apresenta forma ovalada horizontalmente em bovinos, formato de círculo irregular em cabras e aparência de rim em ovelhas (Maggs et al., 2013). Gelatt (2008) afirmou que, em bovinos, o disco óptico está normalmente localizado no fundo não tapetal, logo abaixo da região de junção da área tapetal com a não tapetal, e sua coloração é variada, podendo estar nas cores laranja e marrom. Assim, os resultados obtidos neste estudo foram condizentes com a literatura consultada.

Nos animais avaliados, por serem hígidos, não foram identificadas alterações de tamanho nem de coloração, mas os resultados poderão ser utilizados como fonte de consulta em casos de suspeitas das doenças citadas anteriormente. Em bovinos, o disco óptico com tamanho reduzido pode ser visualizado em casos de atrofia ou aplasia/hipoplasia, enquanto o tamanho aumentado pode ser ocasionado por mielinização excessiva, papiledema devido à compressão das fibras nervosas, ou neurite óptica. A coloração também pode estar alterada, apresentando-se pálida em casos de atrofia e hiperêmica na neurite (Townsend, 2010).

$\mathrm{Na}$ região central do disco óptico, observou-se, em todos os animais, um ponto esbranquiçado, $\mathrm{o}$ qual se associou à papila de Bergmeister, que é o resquício embrionário da artéria hialoide, observada em bovinos na forma de uma pequena projeção que se estende do nervo óptico para a câmara vítrea, como descrito por Pearce e Moore (2013). A borda externa do disco óptico com coloração esbranquiçada tem sido descrita nesta espécie como uma bainha mielina do nervo óptico (Gelatt, 2008), o que explica o contorno esbranquiçado visualizado no disco óptico dos bovinos examinados.

Nos animais avaliados, notou-se a presença de vasos em toda a superfície da retina e de quatro pares de vasos primários, corroborando Gelatt (2008), que descreve o padrão vascular retiniano em bovinos como do tipo holangiótico, formado por vasos que atravessam toda a superfície da retina, e que, normalmente, possui de três a quatro pares de vasos primários. Os vasos sobrejacentes podem ter calibre diminuído em degenerações retinianas ou em anemias graves e calibre aumentado em vasculite ou neurite óptica (Townsend, 2010), o que não foi observado em nenhum dos grupos.

Os valores obtidos no exame de biometria apresentaram diferença entre os grupos GN e GG $(\mathrm{P}<0,05)$ para as medidas do comprimento axial do bulbo ocular, do diâmetro da lente e da profundidade da câmera vítrea. Potter et al. (2008) também observaram para as mesmas medidas diferenças biométricas entre as raças Jersey e Holandesa, por ultrassonografia em modo B, demonstrando que as medidas das estruturas oculares de bovinos foram influenciadas pela raça.

Os resultados da mensuração do comprimento axial ocular dos bovinos Nelore e Gir foram menores que os encontrados por Corrêa et al. (2014) em olhos extraídos de bovinos da raça Nelore $(3,33 \pm 0,15 \mathrm{~cm})$, utilizando-se paquímetro, e por Potter et al. (2008) nas raças Holandesa $(3,46 \pm 0,09 \mathrm{~cm})$ e Jersey $(3,27 \pm 0,19 \mathrm{~cm})$, com ultrassonografia em modo B. Na avaliação de olhos de carcaças de animais da raça Angus $(3,32 \pm 0,01 \mathrm{~cm})$ realizada por El-Maghraby et al. (1995), também foram obtidos valores superiores aos verificados nos animais do presente estudo. A divergência entre os resultados pode ter sido devido às idades dos animais, 30 meses, 66 meses e 24 meses, respectivamente, enquanto os bovinos utilizados neste estudo tinham idade média de 15 meses.

Na avaliação da câmera anterior, não se notaram diferenças significativas entre as raças. Os valores encontrados nos bovinos das raças Nelore e Gir foram maiores quando comparados com os das raça Holandesa $(0,33 \pm 0,05 \mathrm{~cm})$ e Jersey $(0,36 \pm 0,07 \mathrm{~cm})$ (Potter et al., 2008), e menores quando comparados aos valores obtidos em olhos extraídos de animais da raça Angus $(0,62 \pm 0,04 \mathrm{~cm})$ (El-Maghraby et al., 1995). Potter et al. (2008) relataram que pode ocorrer a subestimação da profundidade da câmara anterior devido à depressão da superfície da córnea pela pressão do transdutor, o que pode justificar os valores diferentes, uma vez que cada examinador pode ter inferido pressões diferentes.

As medidas de profundidade da lente aferidas neste estudo foram inferiores aos resultados de Corrêa et al. (2014), que avaliaram bulbos 
oculares extraídos de bovinos da raça Nelore $(1,53 \pm 0,10 \mathrm{~cm})$ por meio de um paquímetro. Da mesma forma, foram observados valores superiores em bovinos das raças Holandesa $(1,78 \pm 0,09 \mathrm{~cm})$ e Jersey $(1,92 \pm 0,11 \mathrm{~cm})$ (Potter et al., 2008) e, também, em olhos extraídos de animais da raça Angus $(1,13 \pm 0,04 \mathrm{~cm})$ (ElMaghraby et al., 1995). Kassab (2012) relatou que a idade influenciou as dimensões da lente em camelos e búfalos. Isso porque o córtex da lente aumenta ao longo da vida, resultado da produção contínua de novas fibras (Corrêa et al., 2014), o que justifica os valores divergentes encontrados neste estudo.

O comprimento da câmera vítrea dos animais da raça Nelore e da raça Gir foi similar aos obtidos por ultrassonografia em modo B em bovinos das raças Holandesa $(1,46 \pm 0,09 \mathrm{~cm})$ e Jersey $(1,62 \pm 0,04 \mathrm{~cm})$ (Potter et al., 2008), bem como em olhos post mortem de bovinos da raça Angus $(1,37 \pm 0,09 \mathrm{~cm})$ (El-Maghraby et al., 1995).

\section{CONCLUSÕES}

Os exames oftalmológicos (teste lacrimal de Schirmer-1, tonometria de aplanação, retinografia e biometria) podem ser realizados a campo, sem comprometerem o bem-estar dos animais de ambas as raças. Comparativamente, os olhos dos bovinos da raça Gir são maiores que os olhos dos Nelores.

\section{REFERÊNCIAS}

ANDRADE, S.F.; KUPPER, D.S.; PINHO, L.F.R. et al. Evaluation of the Perkins handheld applanation tonometer in horses and cattle. $J$. Vet. Sci., v.12, p.171-176, 2011.

BECKWITH-COHEN, B.; ELAD, D.; BDOLAH-ABRAM, T.; OFRI, R. Comparison of tear $\mathrm{pH}$ in dogs, horses, and cattle. Am. J. Vet. Res. v.75, p.494-499, 2014.

BRAEKEVELT, C.R. Fine structure of the bovine tapetum fibrosum. Anat. Histol. Embryol. v.15, p.215-222, 1986.

CORRÊA, L.B.N.S.; SANTA'ANNA JUNIOR, L.P.; SOUZA JUNIOR, P.D. Biometria do bulbo ocular de bovinos da raça nelore. Cienc. Anim. Bras., v.15, p.207-212, 2014.
EL-MAGHRABY, H.M.; NYLAND, T.G.; BELLHORND, D. Ultrasonographic and biometric evaluation of sheep and cattle eyes. Vet. Radiol. Ultrasound, v.36, p.148-151, 1995.

FERREIRA, M.A.; ALLEMANN, N.; DIAS, L.G.G.G.; HONSHO, C.S. Relation between opfthalmic ultrasound biometry and the morphometric parameters of the skull, age, weight and gender in domestic cats. Pesqui. Vet. Bras., v.34, p.192-198, 2014.

GELATT, K.N. Manual de oftalmologia veterinária. São Paulo: Manole, 2003. 594p.

GELATT, K.N. Veterinary ophthalmology: our past, present and future. Bull. Acad. Vét. Fr., v.161, p.299-306, 2008.

GHAFFARI, M.S.; SHOJAEI, M.; SABZEVARI, A.; KHORAMI, N. Reference values for intraocular pressure and Schirmer tear test in clinical normal Sanjabi sheep. Small Ruminant Res. v.97, p.101-103, 2011.

GUM, G.G.; GELATT, K.N.; MILLER, D.N.; MACKAY, E.O. Intraocular pressure in normal dairy cattle. Vet. Ophthalmol., v.1, p.159-161, 1998.

HONSHO, C.S.; JORGE, A.T.; OLIVEIRA, L.T. et al. Intraocular pressure and Schirmer tear test values in maned wolf (Chrysocyon brachyurus). Pesqui. Vet. Bras. v.36, p.919-923, 2016.

KASSAB, A. Ultrasonographic and macroscopic anatomy of the enucleated eyes of the Buffalo (Bos bubalis) and the One-Humped Camel (Camelus dromedarius) of different ages. Anat. Histol. Embryol., v.41, p.7-11, 2012.

MAGGS, D.J.; MILLER, P.E.; OFRI, R. Slatter's fundamentals of veterinary ophthalmology. 5.ed. St. Louis: Elsevier Health Sciences, 2013. 506p.

OLLIVIER, F.J.; SAMUELSON, D.A.; BROOKS, D.E. et al. Comparative morphology of the tapetum lucidum (among selected species). Vet. Ophthalmol., v.7, p.11-22, 2004.

ORIÁ, A.P.; GOMES JUNIOR, D.C.; ARRAES E.A. et al. Tear production, intraocular pressure and conjunctival microbiota, cytology and histology of new zealand rabbits (Oryctolagus cuniculus). Pesqui. Vet. Bras. v.34, p.1024-1028, 2014. 
PEARCE, J.W; MOORE, C.P. Food Animal ophthalmology. In: GELATT, K.N.; GILGER, B.C; KERN, T.J. (Eds.). Veterinary ophthalmology. 5.ed. Iowa: Wiley-Blackwell, 2013. p.1611-1674.

PIETRO, S.; PASSANTINO, A.; CRINÒ, C. et al. Comparison of tear production and intraocular pressure in domestic and wild ruminants. Large Anim. Rev., v.22, p.39-42, 2016.

POTTER, T.J.; HALLOWELL, G.D.; BOWEN, I.M. Ultrasonographic anatomy of the bovine eye. Vet. Radiol. Ultrasound, v.49, p.172-175, 2008.

SANTOS, L.R.H.; CASTRO, P.D.; CASTRO, L.P. et al. Anomalías del desarrollo del segmento anterior. Rev. Cuban. Oftalmol., v.25, p.119-132, 2012.
SARCINELLI, M.F.; VENTURINI, K.S.; SILVA, L.C. Produção de bovinos - tipo leite. Vitória: UFES, 2007. 9p.

TEKTAS, O.Y.; HAMMER, C.M.; DANIAS, J. Morphologic changes in the outflow pathways of bovine eyes treated with corticosteroids. Invest. Ophthalmol. Vis. Sci., v.51, p.4060-4066, 2010.

TOFFLEMIRE, K.L.; WHITLEY, E.M.; GOULD, S.A. et al. Schirmer tear test I and rebound tonometry findings in healthy calves. Vet. Ophthalmol., v.18, p.147-151, 2015.

TOWNSEND, W.M. Examination techniques and therapeutic regimens for the ruminant and camelid eye. Vet. Clin. Food. Anim., v.26, p.437$458,2010$.

WIESER, B.; TICHY, A.; NELL, B. Correlation between corneal sensitivity and quantity of reflex tearing in cows, horses, goats, sheep, dogs, cats, rabbits, and guinea pigs. Vet. Ophthalmol., v.16, p.251-262, 2013. 